\title{
COMMENTARY
}

\section{Should we abandon manual muscle strength testing in the ICU?}

\author{
Greet Hermans ${ }^{1}$ and Rik Gosselink ${ }^{2 *}$ \\ See related research by Hough et al., http://ccforum.com/content/15/1/R43
}

\begin{abstract}
Intensive-care-unit-acquired weakness is a major complication in critically ill patients. The paper by Hough and coworkers suggests that the current method of manual muscle strength testing with the Medical Research Council sum score is of limited value in the intensive care unit. However, their results raise a number of questions and provide important lessons for implementation of such evaluations in the intensive care unit.
\end{abstract}

Intensive-care-unit-acquired weakness (ICUAW) is a major complication in critically ill patients. The paper by Hough and colleagues addresses the feasibility and reliability of manual muscle testing in the intensive care unit (ICU) [1]. ICUAW is associated with prolonged weaning, with delayed rehabilitation and with mortality [2-4]. Early diagnosis is important to warn for weaning failure, to guide progressive ambulation and to predict outcome. Manual muscle strength testing using the Medical Research Council (MRC) sum score was proposed as a diagnostic criterion for ICUAW [5]. Hough and colleagues conclude that the MRC sum score is of limited value in patients in the ICU [1]. So should we now discard manual muscle testing in the ICU?

Previous results on the reproducibility of the MRC sum score in patients with Guillain-Barré syndrome and in a group of post-ICU patients and stimulants showed very good reproducibility [6,7]. The authors legitimately questioned whether these results could be extrapolated to patients in the ICU, as fluctuating consciousness or pain may affect reliability of the measurement. The outcome of the study by Hough and colleagues raises

*Correspondence: rik.gosselink@faber.kuleuven.be

2Department of Rehabilitation Sciences, Katholieke Universiteit Leuven, Herestraat 49, B3000 Leuven, Belgium

Full list of author information is available at the end of the article several issues. Although the study was set up to evaluate the MRC sum score in critically ill patients, only onethird of the results were obtained in the ICU and the majority of patients were studied post ICU. Indeed, decreased consciousness limits the feasibility of the MRC score in the ICU [4,8]. Nevertheless, $28 \%$ [4] to $78 \%$ [8] of patients survived to awakening and were evaluable in the ICU, which is much higher than the 7\% (10/135) reported in Hough and colleagues' paper.

The high reliability of diagnosing ICUAW in the postICU setting confirms previous findings [6]. The very low agreement found by Hough and colleagues in the ICU $(\kappa=0.38)$, however, may be due to several methodological issues.

Firstly, stringent criteria for levels of cooperation were lacking. Fulfillment of only three out of five criteria for adequacy was required. This low number indicates suboptimal cognitive function, and variable response to volitional muscle testing is not surprising.

Secondly, manual muscle testing is a routine clinical examination for skilled physiotherapists. Standardization of this procedure and adaptation to the specific situation of the bed-ridden ICU patients is crucial. Body positioning and the limb starting position for the tested muscle groups are potential causes of bias. Hough and colleagues measured patients in the sitting or supine position and did not provide information on limb positioning. Criteria to differentiate between two scoring levels, such as the required range of motion against gravity to obtain a score of 3 , should be specified. Other determinants are the hand position while applying resistance, encouragement and the assessor's experience and muscle strength. Failure to control these factors could have contributed to the poor agreement.

Thirdly, the muscle strength reported in this study is surprisingly high, with a median value of 55 (interquartile range 49 to 58) and of 56 (interquartile range 50 to 58 ) for both observers, and an incidence of $17 \%$ for ICUAW in a population with a median duration of mechanical ventilation of 10 days. These results are in contrast with previous studies showing an incidence of $25 \%$ in patients ventilated for at least 5 days [8] to 7 days [4], which may 
be due to the predominance of measurements post ICU, during recovery. Median values for the individual muscle groups are $\geq 4.5$ in 10 out of 12 muscle groups. The low inter-rater reliability reported for the individual muscle groups may therefore mainly reflect the difficulty in differentiating between score 4 and score 5 [9]. Finally, it is notable that the sample size was very small.

Although reproducibility of the MRC sum score in the ICU was not formally tested before, its relevance in the ICU is clear. The score was successfully implemented in the ICU in several studies showing its relationship with respiratory muscle force, weaning and mortality $[4,8,10]$. Evaluation of the MRC sum score is restricted to cooperative patients. It is questionable whether a diagnosis of ICUAW is relevant in patients who do not regain consciousness in the ICU [4]. The MRC sum score, in contrast with nonvolitional evaluations such as electrophysiology and magnetic stimulation elicited contractions, has the advantage of being easy to perform at the bedside, cheap and potentially widely available. The paper by Hough and colleagues underscores the caution that is needed when the score is implemented in the ICU.

In conclusion, these data highlight the need to study reproducibility of manual muscle testing in ICU patients. Further study should include rigid criteria to judge consciousness and cooperation that reliably allow prediction of successful comprehension to perform 12 consecutive tasks, and using clear and detailed protocols adapted for bed-ridden patients. In addition, studying the muscle strength of patients over the full range of the MRC sum score will allow one to judge the reliability in each grade of this score. The population studied should be large enough to answer these questions and to allow firm recommendations towards specific requisites for reliable use of the MRC sum score in the ICU.

\section{Abbreviations}

ICU, intensive care unit; ICUAW, intensive-care-unit-acquired weakness; MRC, Medical Research Council.
Competing interests

The authors declare that they have no competing interests.

\section{Author details}

'Medical Intensive Care Unit, Department of General Internal Medicine, University Hospitals Leuven, Belgium. ${ }^{2}$ Department of Rehabilitation Sciences, Katholieke Universiteit Leuven, Herestraat 49, B3000 Leuven, Belgium.

Published: 1 March 2011

\section{References}

1. Hough CL, Lieu BK, Caldwell ES: Manual muscle strength testing of critically ill patients: feasibility and interobserver agreement. Crit Care 2011, 15:R43.

2. Stevens RD, Dowdy DW, Michaels RK, Mendez-Tellez PA, Pronovost PJ, Needham DM: Neuromuscular dysfunction acquired in critical illness: a systematic review. Intensive Care Med 2007, 33:1876-1891.

3. Leijten FS, Harinck-de Weerd JE, Poortvliet DC, de Weerd AW: The role of polyneuropathy in motor convalescence after prolonged mechanical ventilation. JAMA 1995, 274:1221-1225.

4. De Jonghe B, Sharshar T, Lefaucheur JP, Authier FJ, Durand-Zaleski I, Boussarsar M, Cerf C, Renaud E, Mesrati F, Carlet J, Raphael JC, Outin H, Bastuji-Garin S: Paresis acquired in the intensive care unit: a prospective multicenter study. JAMA 2002, 288:2859-2867.

5. Stevens RD, Marshall SA, Cornblath DR, Hoke A, Needham DM, De JB, Ali NA, Sharshar T: A framework for diagnosing and classifying intensive care unit-acquired weakness. Crit Care Med 2009, 37:S299-S308.

6. Fan E, Ciesla ND, Truong AD, Bhoopathi V, Zeger SL, Needham DM: Inter-rater reliability of manual muscle strength testing in ICU survivors and simulated patients. Intensive Care Med 2010, 36:1038-1043.

7. Kleyweg RP, van der Meche FG, Schmitz PI: Interobserver agreement in the assessment of muscle strength and functional abilities in Guillain-Barre syndrome. Muscle Nerve 1991, 14:1103-1109.

8. Ali NA, O'Brien JM Jr, Hoffmann SP, Phillips G, Garland A, Finley JC, Almoosa K, Hejal R, Wolf KM, Lemeshow S, Connors AF Jr, Marsh CB: Acquired weakness, handgrip strength, and mortality in critically ill patients. Am J Respir Crit Care Med 2008, 178:261-268.

9. Bohannon RW: Measuring knee extensor muscle strength. Am J Phys Med Rehabil 2001, 80:13-18.

10. De Jonghe B, Bastuji-Garin S, Durand MC, Malissin I, Rodrigues P, Cerf C, Outin $H$, Sharshar T: Respiratory weakness is associated with limb weakness and delayed weaning in critical illness. Crit Care Med 2007, 35:2007-2015.

doi:10.1186/cc10038

Cite this article as: Hermans G, Gosselink R: Should we abandon manual muscle strength testing in the ICU? Critical Care 2011, 15:127. 